\title{
Une analyse formelle du jeu des acteurs autour du projet de barrage de Sivens
}

\author{
Christophe Sibertin-Blanc* \\ Informatique, IRIT, Université Toulouse 1 Capitole, Toulouse, France
}

Reçu le 5 mai 2016. Accepté le 27 novembre 2017

À partir d'un cas bien documenté, le projet de barrage de Sivens, en se basant sur la sociologie de l'action organisée de Michel Crozier et Erhard Friedberg, l'auteur propose une modélisation du conflit. Les données sont mesurées sur des échelles ordinales à partir d'entretiens par questionnaire pour évaluer les degrés de satisfaction des différents acteurs et les poids des différentes contraintes. Certes, la structure du système est très particulière et linéaire, entraînant une "surdétermination" des comportements qui conduit au blocage et à l'affrontement. Pourtant, une «analyse de sensibilité» indique que d'autres positionnements auraient pu conduire à un relâchement dans la radicalité des oppositions. Utilisée en modélisation d'accompagnement, cette approche qui décrypte et explicite les comportements des acteurs a pour visée d'imaginer des solutions ouvrant sur un dialogue entre acteurs.

La Rédaction

Résumé - Le pays a été stupéfait d'apprendre la mort d'un opposant à la construction d'un barrage dans la forêt de Sivens, dans le Tarn, lors d'affrontements avec les forces de l'ordre dans la nuit du 25 au 26 octobre 2014. Pourtant, l'importance des moyens déployés pour réaliser cet ouvrage et la détermination des opposants, conjuguées au jeu de l'ensemble des parties prenantes de ce projet, laissaient présager la possibilité d'un tel drame. À l'aide d'une grille d'analyse formelle fondée sur la sociologie de l'action organisée, nous présentons un modèle de ce jeu d'acteurs dont les résultats de simulation mettent en évidence le caractère surdéterminé de l'émergence d'un conflit d'une extrême intensité. Des variations de ce modèle permettent ensuite de dégager les principaux déterminants de ce conflit et d'envisager un autre futur possible.

Mots-clés : environnement / gouvernance / conflits d'usage / modèle formel / simulation sociale

\begin{abstract}
A formal analysis of the interplay of actors involved in the Sivens dam project. The French public was stunned by the death of an opponent to the construction of a dam in the Sivens forest (southwestern France) during confrontations with the police on the night of 25 to 26 October 2014. Yet the considerable means deployed to carry out this project and the determination of opponents, embedded in the interactions between all the stakeholders of this project, foreshadowed the possibility of this exceptional drama. We have used a formal analysis framework based on the sociology of organized action to present a model of this interaction system: the main actors, their respective means of action and their dependence on each other to reach their objectives. The structural analysis of this model highlights the position of each actor and the main conflicts between them. A bounded rationality algorithm, which measures how each actor learns to develop a behavior that enables him/her to reach their objectives in the best possible way according to the behavior of others, allows to make simulations whose results show the overdetermined emergence of a clash of extreme intensity. Variations in this model then make it possible to identify the main determinants of this conflict and to consider another possible future.
\end{abstract}

Keywords: environment / governance / conflicts of use / formal models / social simulation

\footnotetext{
*Auteur correspondant : sibertin@ut-capitole.fr
} 
Le projet de barrage dans la forêt de Sivens, initié par le conseil général du Tarn en 2007, consistait à réaliser une retenue de 1,5 million de $\mathrm{m}^{3}$ sur le cours du Tescou, un affluent du Tarn dans le bassin de la Garonne, principalement pour l'irrigation de terres agricoles (à hauteur de $70 \%$ ) et le soutien de l'étiage (30\%), pour un coût de $8,4 \mathrm{M} €$. Ce projet a suscité une forte contestation, car il impliquait la disparition de 18 hectares d'une zone humide reconnue « d'importance majeure du département du point de vue de la biodiversité » (GéoDiag et Ecogéa, 2007 ; Scop Sagne, 2010), alors que, étant surdimensionné, il ne constituait pas une «solution adaptée » (Forray et Rathouis, 2014 ; Forray et Roche, 2015). Cette opposition s'est manifestée de deux façons différentes: par la constitution en juin 2012 d'un Collectif qui a acquis une forte expertise sur tous les aspects du projet et a tenté, sans succès, de faire reconnaître ses insuffisances auprès des décideurs et des tribunaux; et par l'occupation du site par des « zadistes ${ }^{1}$ », pour empêcher en 2013 la réalisation des travaux préparatoires (repérage, prélèvement d'espèces protégées, etc.) et en 2014 le déboisement. Ce dernier a eu lieu du $1^{\mathrm{er}}$ au 20 septembre 2014. Durant cette période, les occupants se sont efforcés de l'empêcher (s'installant dans les arbres, s'ensevelissant sur le trajet des engins, etc.) mais l'appui des forces de maintien de l'ordre a permis aux bûcherons de réaliser le déboisement. Le bétonnage de la digue devant commencer le lundi 27 octobre, une coordination des opposants organise le 25 un rassemblement auquel plusieurs milliers de personnes participent.

Dans la nuit du 25 au 26 octobre 2014, un opposant au projet, Rémi Fraisse, est tué par une grenade offensive lors d'affrontements entre les forces antiémeutes et un groupe d'opposants. Cet événement, exceptionnel en France, a soulevé une vive émotion dans le pays et conduit à l'abandon du projet sans que, sur le terrain, les passions ne s'apaisent. Les violences se sont poursuivies jusqu'à l'expulsion des zadistes du site le 6 mars 2015, date à laquelle le conseil général du Tarn a pris «acte de l'impossibilité de poursuivre toute activité liée au déroulement du chantier » tout en décidant d'un nouveau projet de «retenue d'eau pour l'agriculture, redimensionnée sur l'emprise du site». L'accès au site, encore interdit fin 2015, a été surveillé jusque fin 2016. Le «protocole transactionnel ${ }^{2}$ » entre l'État et le conseil général, préalable à l'élaboration d'un nouveau projet, n'a été conclu que le 11 décembre 2015. L'annulation par

\footnotetext{
$\overline{{ }^{1} \text { Appellation donnée aux }}$ personnes occupant les «zones à défendre ».

${ }^{2}$ Annoncé par Ségolène Royal le 27 février 2015, ce protocole stipule que 1) le conseil général renonce à ce projet, 2) le conseil général n'attaque pas l'État pour l'annulation des arrêtés ayant autorisé les travaux, et 3) l'État indemnise le conseil général à hauteur de 3,3 M€.
}

le tribunal administratif de Toulouse de tous les arrêtés autorisant la construction du barrage a donné raison aux opposants le 30 juin 2016. Le projet initial étant définitivement soldé, le site de la zone humide a été réhabilité (octobre 2017) et le préfet du Tarn a initié le 14 novembre 2016 le processus de construction du projet de territoire (toujours en cours fin 2017) ${ }^{3}$.

\section{Comment a-t-on pu en arriver là ?}

Dans la presse et ailleurs, de nombreuses personnes ont témoigné (Collectif, 2014 ; Collectif, 2015), cherché à comprendre ce qui a pu se passer (Foissac, 2015) ou analysé les dysfonctionnements de l'élaboration de ce projet (Souchay et Laimé, 2015; Lefetey, 2015); le Parlement et la Ligue des droits de l'homme ont mené des commissions d'enquête... Sur le moteur de recherche Google, le mot «Sivens » donne lieu à 517000 résultats (le $1^{\text {er }}$ juin 2015).

Courant septembre 2014, plusieurs voix ont lancé des alertes: les moyens déployés sur le site par les forces de l'ordre sont disproportionnés et la violence des affrontements est de nature à provoquer un accident grave (Foissac, 2015 ; Bès et al., 2015). Ces avertissements conduisent à penser que la mort de Rémi Fraisse n'est pas un événement fortuit mais le résultat malheureusement prévisible de la logique des acteurs parties prenantes de ce projet: dans ce processus initié en 2007, le comportement de chacun a conduit, en septembre 2014, le conflit à un niveau d'intensité qui permettait d'avertir de la possibilité d'un drame.

Cet article se propose d'analyser les conditions de l'apparition, en fait la production, d'un tel niveau de conflit par la modélisation des représentations et stratégies des acteurs, qui ont déterminé les comportements que ceux-ci ont adoptés les uns vis-à-vis des autres et qui ont conduit au drame. Par modèle, nous entendons une description quantifiée du système constitué des acteurs, de leurs moyens d'action et de leurs dépendances mutuelles. Un tel modèle est nécessairement réducteur afin de rendre intelligible la complexité des interactions entre les acteurs, mais le caractère simplificateur de cette réduction n'empêche pas de rendre compte fidèlement des aspects essentiels du jeu des acteurs. Un tel modèle échappe à la subjectivité du langage naturel et peut servir de support à la confrontation entre des analyses divergentes des événements: si l'on s'accorde sur la sémantique du modèle, c'est-à-dire sur l'interprétation, dans les termes du cas considéré, de la valeur des variables numériques, les points de désaccord seront clairement établis comme des différends portant sur certains éléments du modèle. Enfin, par des calculs et la

\footnotetext{
${ }^{3}$ On pourra trouver le compte rendu de tous ces épisodes dans La Dépêche du Midi et Le Tarn libre.
} 
Encadré 1. Le méta-modèle SocLab des systèmes d'action organisée.

La structure d'un SAO (Fig. 1) est constituée :

- d'un ensemble «d'acteurs», individus ou collectifs qui disposent chacun de moyens d'action relatifs à l'objet du système ;

- d'un ensemble de «relations» qui constituent le support des interactions entre les acteurs.

Chaque relation est «contrôlée» par un acteur et chaque acteur «dépend» d'un certain nombre de relations pour la réalisation de ses objectifs.

Une relation est fondée sur une (ou plusieurs) ressource matérielle ou cognitive (information, connaissance ou maîtrise de règles, intervention auprès de tiers, autorisation, attitude, etc. [Grossetti, 2004]) utile à d'autres et que maîtrise l'acteur qui la contrôle; cette maîtrise lui confère un moyen d'action sur les acteurs qui en dépendent, et l'«état» d'une relation modélise le comportement adopté par l'acteur dans la gestion de la ressource. Cet état sera plus ou moins bénéfique pour chacun des acteurs qui en dépendent ; évalué sur une échelle de valeur de -10 (très pénalisant) à +10 (très favorable), il caractérise le niveau de coopération de celui qui contrôle la ressource vis-à-vis de l'ensemble des autres acteurs du système.

L' «enjeu» qu'un acteur place sur une relation est fonction de l'importance de la ressource sous-jacente pour la réalisation de ses objectifs : plus l'accès à une ressource est nécessaire pour la réalisation d'un objectif important, plus l'enjeu de l'acteur sur la relation associée sera important. Les enjeux sont quantifiés sur une échelle de 0 (nul) à +10 (capital) et la somme des enjeux de chaque acteur est normalisée à 10. L' «effet» d'une relation sur un acteur est une fonction qui détermine dans quelle mesure, en fonction de son état, cette relation contrarie ou facilite la réalisation des objectifs de cet acteur.

L'effet d'une relation sur un acteur est évalué sur une échelle allant de -10 (pire) à +10 (optimal); il caractérise la contribution de cette relation, selon son état, à la réalisation des objectifs de cet acteur.

Enfin, un acteur peut entretenir certaines « solidarités », personnelles ou organisationnelles, avec d'autres acteurs, ce qui se traduit par la prise en compte, dans l'évaluation de sa propre situation, de celle de ces autres acteurs. Ces solidarités s'expriment sur une échelle allant de -1 (complète hostilité) à 0 (indifférence) et +1 (complète adhésion).

Nous appelons «configuration» d'un SAO la donnée du comportement de chacun des acteurs, c'est-à-dire l'état de chacune des relations. Une configuration procure à chacun d'eux une certaine «satisfaction » qui évalue dans quelle mesure les objectifs de cet acteur sont atteints, ou du moins rendus accessibles, du fait du comportement de l'ensemble des acteurs. Lorsqu'un acteur $a$ n'entretient de solidarité qu'avec lui-même, sa satisfaction dans une configuration $s=\left(s_{r}\right)_{r \in R}$ du SAO s'exprime sous la forme :

$\operatorname{satisfaction}(a, s)=\Sigma_{r \in R}$ enjeu $(a, r) *$ effet $\left(a, s_{r}\right)$

où $R$ est l'ensemble des relations, enjeu $(a, r)$ l'enjeu de l'acteur $a$ sur la relation $r$, et effet $_{r}\left(a, s_{r}\right)$ l'effet sur $a$ de la relation $r$ lorsqu'elle est dans l'état $s_{r}$. La valeur de la satisfaction d'un acteur appartient donc à un intervalle compris entre - 100 et +100 .

Dans le cas général, la satisfaction d'un acteur $a$ prend la forme:

satisfaction $(a, s)=\Sigma_{b \in A}$ Solidarité $(a, b) * \Sigma_{r \in R}$ enjeu $(b, r) *$ effet $_{r}\left(b, s_{r}\right)$

où $A$ est l'ensemble des acteurs et solidarité $(a, b)$ la solidarité de $a$ pour l'acteur $b$.

De façon duale, on peut quantifier le «pouvoir» exercé par un acteur dans une configuration donnée comme la somme de ses contributions, par l'intermédiaire des relations qu'il contrôle, à la satisfaction des autres acteurs, sous la forme (lorsque chaque acteur n'entretient de solidarité qu'avec lui-même):

pouvoir $(a, s)=\Sigma_{r \in R ;}$ a controls $r \Sigma_{b \in A}$ enjeu $(b, r) *$ effet $r\left(b, s_{r}\right)$

réalisation de simulations, un tel modèle permet de produire des résultats susceptibles de mettre en lumière les principaux déterminants et certaines caractéristiques de la structure du système (Axelrod, 1997; Gilbert, 2004).

Dans la prochaine section de cet article, nous présentons la grille d'analyse et les outils que nous utilisons pour élaborer et étudier ce modèle. Dans les deux sections suivantes, nous nous focalisons sur le modèle lui-même: le repérage des acteurs et de leurs moyens d'action puis les quantifications qui leur sont associées. Nous mettons ensuite en évidence quelques propriétés structurelles de ce modèle, et les simulations qui montrent le caractère inéluctable d'un niveau maximal de confrontation. Enfin, nous considérons des évolutions dans la stratégie des acteurs qui conduisent à un modèle dans lequel le niveau de conflit reste circonscrit, et qui, ainsi, mettent en évidence les déterminants principaux de l'occurrence du drame. En conclusion, nous identifions le caractère paradigmatique $\mathrm{du}$ projet de barrage de Sivens et synthétisons la démarche de modélisation ${ }^{4}$.

${ }^{4} \mathrm{La}$ recherche rapportée dans cet article n'a fait l'objet d'aucun financement spécifique et les résultats n'engagent que leur auteur. 


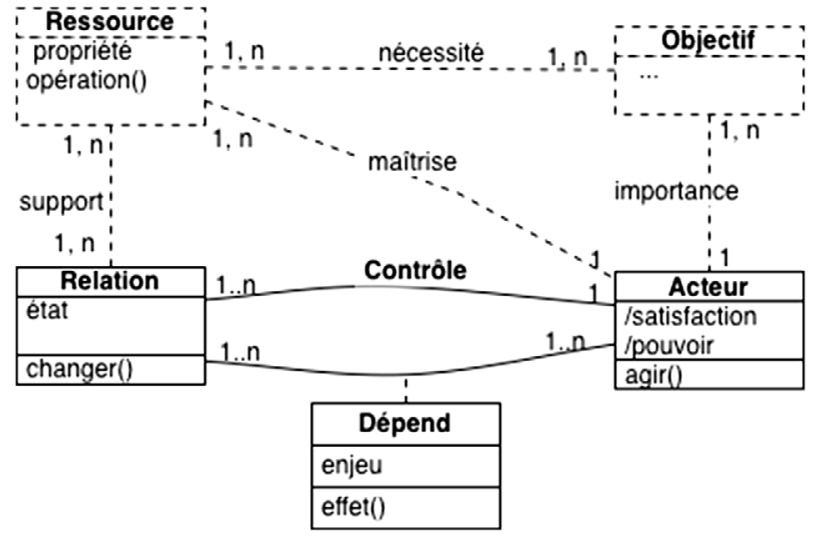

Fig. 1. La structure d'un système d'action organisée, sous la forme d'un diagramme de classe UML (Unified modeling language). Source : auteur.

\section{Le modèle SocLab d'un système d'action organisée}

On entend par système d'action organisée (SAO) le jeu d'acteurs qui interagissent dans un contexte organisationnel plus ou moins bien défini (une entreprise, une association, un système politique, etc.) ou de façon plus diffuse autour d'un objet dont ils sont parties prenantes. L'approche SocLab pour la modélisation et l'étude des SAO est fondée sur la sociologie de l'action organisée développée par Michel Crozier et Erhard Friedberg (Crozier, 1963; Crozier et Friedberg, 1977); une présentation détaillée en est faite dans Sibertin-Blanc et al. (2013) et Adreit et al. (2014).

Le logiciel SocLab ${ }^{5}$ permet d'étudier un SAO en définissant ses éléments constitutifs (sa structure), en calculant des indicateurs sur des caractéristiques structurelles du système d'action et sur les comportements potentiels des acteurs, en explorant l'espace de ses configurations (ce que chacun pourrait faire in abstracto) et en réalisant des simulations qui indiquent comment les acteurs sont concrètement susceptibles de se comporter les uns vis-à-vis des autres (Encadré 1).

La structure du modèle d'un SAO définit les règles d'un jeu d'acteurs dans lequel les acteurs échangent leur comportement par l'intermédiaire de l'effet des relations qu'ils contrôlent: lorsqu'un acteur modifie l'état d'une relation qu'il contrôle, il augmente ou diminue (selon l'orientation des fonctions d'effet) sa contribution à la satisfaction des acteurs qui en dépendent. Selon une hypothèse de rationalité des acteurs, chacun cherche à réaliser ses objectifs du mieux possible, ce qui revient à obtenir la satisfaction la plus élevée possible. Chacun va donc tester différents comportements, afin d'en trouver un qui incite les autres acteurs à lui donner

\footnotetext{
$\overline{{ }^{5} \mathrm{https}: / / \text { soclabproject.wordpress.com. }}$
}

un bon niveau de satisfaction, jusqu'à ce que soit atteinte une configuration d'équilibre dans laquelle aucun ne voit plus comment il pourrait accroître sa satisfaction. Ce jeu donne donc lieu à un apprentissage Collectif au cours duquel chacun cherche comment il doit se comporter pour réaliser au mieux ses objectifs, jusqu'à l'obtention d'une configuration du jeu qui procure à chacun un niveau de satisfaction qu'il accepte. Alors, les comportements des acteurs s'équilibrent les uns les autres et le système d'action peut fonctionner durablement ainsi, dans une configuration qui est régulée.

Le logiciel SocLab comporte un algorithme de simulation de cet apprentissage collectif. Fondé sur la rationalité limitée des acteurs (Simon, 1982), il calcule des configurations soutenables, socialement plausibles. Il est présenté en détail dans El Gemayel et al. (2011) et Sibertin-Blanc et El Gemayel (2013).

Le modèle du système d'action organisée autour du projet de barrage de Sivens présenté ici repose sur les dires de participants à ce système d'action que nous avons interrogés au printemps et à l'automne 2015, les données quantitatives étant consignées au moyen de la grille d'entretien figurant en annexe. Nous étayerons ce modèle par les éléments qui nous semblent les plus significatifs, l'ensemble du projet de barrage de Sivens étant par ailleurs très bien documenté.

\section{Les acteurs du modèle}

Commençons par identifier les acteurs concernés par la réalisation du barrage de Sivens, nous étudierons ensuite la quantification de leurs dépendances mutuelles. Sur la période 2007-2015, le système d'action autour du projet de barrage a évolué, du fait de la succession des titulaires du ministère de l'Écologie mais aussi, de façon endogène, du fait de l'évolution de la représentation des acteurs. Le modèle que nous présentons ici correspond à la situation qui, depuis le ré-investissement du site par les zadistes le 15 août 2014, prévalait au moment du drame.

Quelques éléments du contexte national et régional dans lequel s'inscrit ce projet de barrage éclairent les enjeux. Devant la virulence des oppositions à la réalisation de l'aéroport de Notre-Dame-des-Landes, près de Nantes, l'État a décidé en mai 2014 de suspendre toutes les opérations en cours jusqu'à l'épuisement des recours en justice déposés par les opposants, soit pour une durée de deux années ${ }^{6}$. C'est à cette occasion qu'est apparue la

\footnotetext{
${ }^{6}$ Dans le même registre, en octobre 2014 l'État «suspendait» l'écotaxe suite aux violentes manifestations des «bonnets rouges » (Libération, 10/10/2014). Plus généralement, voir «Le durcissement des conflits environnementaux» (Le Monde, 07/08/2015). L'État renoncera définitivement à ce projet d'aéroport en janvier 2018.
} 
Tab. 1. Les acteurs du modèle, regroupements d'acteurs du terrain.

\begin{tabular}{lll}
\hline Acteur du modèle & Acteurs de terrain & Relation contrôlée \\
\hline État & Ministres concernés, forces de l'ordre, préfet & soutien \\
CG & $\begin{array}{l}\text { Conseil général Tarn et Tarn-et-Garonne, politiques locaux, milieux } \\
\text { agricoles, Avet }\end{array}$ & engagement \\
CACG & $\begin{array}{l}\text { Compagnie d'aménagement des coteaux de Gascogne } \\
\text { Collectif }\end{array}$ & $\begin{array}{l}\text { Collectif Testet, mouvance écologiste (associations, syndicat, EELV), } \\
\text { comités d'experts }\end{array}$ \\
Zadistes & Visiteurs et occupants du site & expertise \\
\hline
\end{tabular}

Source : auteur.

notion de ZAD ( «zone d'aménagement différé » instituée en «zone à défendre») à l'encontre de «grands projets imposés et inutiles», mouvement émergent de la contestation des grands projets d'aménagement dans lequel les occupants de Sivens se sont inscrits. Courant 2014, l'État était confronté à d'autres projets remettant en cause sa légitimité (Subra, 2014; Milanesi et al., 2018).

En application de la directive cadre sur l'eau de la Communauté européenne ${ }^{7}$, la loi sur l'eau et les milieux aquatiques ${ }^{8}$ a instauré de nouvelles règles pour le calcul et la répartition des volumes prélevables pour l'irrigation. Le bassin Adour-Garonne connaît annuellement un déficit structurel de l'ordre de 250 millions de $\mathrm{m}^{3}$ (Marcaillou, 2014) ${ }^{9}$, alors que l'agriculture représente $11 \%$ des emplois dans le département du Tarn et 7,1\% dans le Tarn-et-Garonne ${ }^{10}$. Dans le bassin Adour-Garonne, l'instauration de ces nouvelles dispositions a donné lieu à un conflit très dur entre la profession agricole et les pouvoirs publics qui a conduit à un protocole d'accord, entre l'État et les chambres d'agriculture de la région, totalement contradictoire avec la réforme des volumes prélevables (Souchay et Laimé, 2015).

Le rapport de la Cour des comptes (2015) concernant les agences de l'eau préconise davantage de transparence et de sélectivité dans l'attribution des aides et de «mettre en place un dispositif de prévention des conflits d'intérêt

\footnotetext{
${ }^{7}$ Water Framework Directive. Directive 2000/60/EC of the European Parliament and of the Council of 23 October 2000 establishing a framework for Community action in the field of water policy, UE.

${ }^{8}$ LEMA, loi n ${ }^{\circ} 2006-1772$ du 30 décembre 2006 sur l'eau et les milieux aquatiques. JORF $\mathrm{n}^{\circ} 303$, p. $20285 \mathrm{sq}$.

${ }^{9}$ La capacité cumulée de soutien d'étiage en service dans le bassin Adour-Garonne est passé de 99 millions de $\mathrm{m}^{3}$ en 1984 à 464 millions de $\mathrm{m}^{3}$ en 2009 (réserves de plus de 2 millions de $\mathrm{m}^{3}$ ) pour un coût de $814 \mathrm{M} €$ (données de l'agence de l'eau Adour-Garonne).

${ }^{10}$ Données 2012 de la chambre régionale d'agriculture de Midi-Pyrénées.
}

pour les membres des instances de gouvernance des agences et pour leur personnel ». En l'occurrence, les mêmes personnes siègent dans les conseils généraux, à l'agence de l'eau Adour-Garonne et à la compagnie d'aménagement des coteaux de Gascogne (CACG). Même si le financement du barrage de Sivens n'a donné lieu à aucune faute individuelle, cette logique de légitimations croisées instaure un entre soi défavorable à l'écoute des points de vue divergents (Lefetey, 2015).

Le système d'action qui s'est noué autour du projet de réaliser un barrage sur le Tescou dans la forêt de Sivens comporte un grand nombre d'acteurs, tant du fait de la complexité institutionnelle d'un pays fortement administré comme la France que de la diversité des modes d'expression de la «société civile». Cependant, l'analyse des intérêts, enjeux, effets des actions des différents acteurs sur les stratégies des autres permet de repérer des convergences qui autorisent le regroupement de plusieurs acteurs du terrain en un seul acteur du modèle; nous y reviendrons plus loin. Il en résulte une simplification du jeu qui ne le dénature pas et améliore son intelligibilité.

Le modèle que nous présentons se focalise sur la survenue de l'accident mortel résultant de l'affrontement entre certains des opposants au barrage et les forces de l'ordre. Organisé autour de ces deux acteurs de terrain, ce modèle comporte cinq acteurs : l'État (le régulateur), le conseil général du Tarn (CG, le maître d'ouvrage), la CACG (le maître d'œuvre), le Collectif Testet (opposant légaliste) et les zadistes (opposant activiste). Chaque acteur contrôle une seule relation qui repose sur les moyens d'action, ou ressources, qu'il maîtrise (Tab. 1).

\section{L'État}

Par État, nous entendons les ministres qui sont directement intervenus dans le jeu: le ministre de l'Écologie et du Développement durable-en l'occurrence Delphine Batho (jusqu'en juin 2013) puis Philippe Martin, Ségolène Royal nommée en mai 2014 ne s'étant 
saisie que tardivement du dossier-et le ministre de l'Intérieur, soutenus par le ministre de l'Agriculture et le Premier ministre. Nous entendons aussi le directeur départemental de la sécurité publique et les forces de l'ordre sur lesquelles il a autorité (en l'occurrence gendarmes mobiles, PSIG et CRS). Le préfet du Tarn (Josiane Chevalier jusqu'au 30 août 2014 puis Thierry Gentilhomme) est le représentant de l'État sur le terrain.

Quels sont les moyens d'action de l'État? D'une part la délivrance des autorisations nécessaires à la réalisation du barrage et d'autre part l'engagement des forces de l'ordre pour protéger la réalisation des travaux. Si ces moyens d'action sont de natures bien différentes, ils ont été mis en jeu par l'État de façons convergentes avec des effets similaires sur les autres acteurs, si bien que leur dissociation compliquerait inutilement le modèle. L'acteur État contrôle donc une seule relation, "soutien", dont les valeurs négatives correspondent à un contrôle strict de la légalité et une certaine tolérance vis-à-vis de l'occupation de la zone de travaux par les zadistes, et les valeurs positives aux comportements inverses.

\section{Le conseil général du Tarn, les pouvoirs locaux et les agriculteurs pro-barrage}

Le conseil général du Tarn est le leader de la coalition des "pro-barrage »: maître d'ouvrage de ce projet initié en $2007^{11}$ et définitivement adopté par la commission permanente du département en mai 2013, il le finance à hauteur de $10 \%$, tout comme le CG du Tarnet-Garonne. Ce projet correspond à un projet remontant à 1978, réactivé par un nouveau rapport de la CACG (2001) indiquant un besoin de 1,5 million de $\mathrm{m}^{3} \mathrm{~d}$ 'eau et rendu possible par l'acquisition de la forêt de Sivens (1976) et de deux exploitations agricoles limitrophes (en 1997 puis en 2002). Le conseil général du Tarn-etGaronne, où est située la vingtaine des principaux bénéficiaires du projet pratiquant la culture de maïs semence à haute rentabilité, est lui aussi très attaché à la réalisation de ce barrage, en conformité avec son soutien à l'agriculture productiviste. Il en est de même des élus locaux, tels la maire de Lisle-sur-Tarn (nouvellement élue en 2014), commune sur laquelle le barrage doit être construit, le député ou l'association des maires et élus du Tarn. La Fédération nationale des syndicats d'exploitants agricoles (FNSEA) et son instance dans le Tarn (FDSEA), ainsi que les Jeunes Agriculteurs et la Coordination rurale, sont des syndicats professionnels agricoles qui mobilisent les agriculteurs (Callon, 1986) pour demander la réalisation de barrages (Marcaillou, 2014), et sont majoritaires au sein de la chambre

\footnotetext{
${ }^{11}$ Le 10 février, avis d'appel d'offre pour «la concession d'aménagement du barrage de Sivens».
}

d'agriculture du Tarn ${ }^{12}$. La politique mise en œuvre par le $C G$ est (conforme à) celle qu'ils souhaitent voir adoptée. Enfin, cette coalition est épaulée par l'Avet (association Vie Eau Tescou), qui a suscité, en marge des enjeux sur la réalisation du barrage, la constitution d'une véritable milice qui ne supporte pas la présence et le mode de vie des zadistes ${ }^{13}$.

Les moyens d'action du conseil général du Tarn sont la communication, dans la presse et auprès de la population, à l'occasion des actes administratifs nécessaires à la réalisation des travaux, notamment les ordres de travaux pour le maître d'œuvre, la CACG. Les moyens d'action des syndicats agricoles sont le soutien (manifestations, presse, tracts, etc.), aux niveaux national et local, à la réalisation du barrage, occasionnellement accompagné par l'Avet ${ }^{14}$. Les moyens de l'Avet sont des opérations commando et d'intimidation in situ. Ces divers moyens d'action ont été mis en œuvre conjointement dans le même sens, ce qui nous autorise à les regrouper dans une seule relation, "engagement». Ses valeurs positives correspondent à la mise en œuvre de ces moyens pour la réalisation du barrage et à l'encontre des opposants, ses valeurs négatives au refus du barrage.

\section{La compagnie d'aménagement des coteaux de Gascogne (CACG)}

La CACG est le maître d'ouvrage délégué du projet depuis 2009 par convention de concession d'aménagement et le très probable gestionnaire du futur barrage, mais c'est en tant que maître d'œuvre de sa construction que nous le considérons ici. La CACG est une société d'économie mixte de deux cents salariés dédiée «à l'aménagement, à l'équipement et au développement économique des régions Midi-Pyrénées et Aquitaine» ayant notamment pour mission la maîtrise de l'eau. C'est le seul opérateur important de ce type en Midi-Pyrénées. La CACG s'est intéressée à la réalisation d'une retenue sur le Tescou dès 1983 (Lefetey, 2015). C'est la CACG qui a établi, à la suite d'un avant-projet en 1989, le

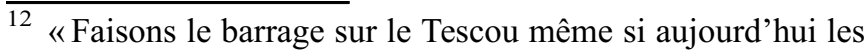
règles font que ce n'est plus possible », interview de J-C. Huc, président de la chambre d'agriculture du Tarn, La Dépêche $d u$ Midi, 04/11/2014.

${ }^{13}$ Voir par exemple Foissac (2015) et «Menace sur les zadistes » (Le Canard enchaîné, 04/02/2015). Bès et al. (2015) analysent bien l'antagonisme fondamental entre les représentations des tenants et des opposants au barrage.

${ }^{14} \hat{A}$ ce titre, les syndicats agricoles pourraient aussi être associés à l'État, tant du fait des relations étroites entre la FNSEA et le ministère de l'Agriculture que de la complaisance coutumière des forces de l'ordre vis-à-vis des dégradations et violences opérées par certains agriculteurs (et l'Avet en l'occurrence).
} 
rapport de 2001 prônant, sans autre alternative, un barrage. Les coûts et le surdimensionnement de celui-ci ont été relevés ultérieurement par les experts mandatés par Ségolène Royal (Forray et Rathouis, 2014 ; Forray et Roche, 2015). Ce rapport a servi de base au plan de gestion des étiages (PGE) du Tescou, adopté en 2004 sous la maîtrise d'ouvrage du CG du Tarn-et-Garonne; de ce fait, ce projet de barrage figure au programme de mesures du schéma directeur d'aménagement et de gestion des eaux (SDAGE) 2010-2015 ${ }^{15}$ du bassin Adour-Garonne, ce qui a conduit à son financement par l'agence de l'eau. Ce rapport, actualisé en 2009, n'a été accessible qu'en juillet 2013, suite à une saisine de la commission d'accès aux documents administratifs (CADA) par le Collectif Testet; il ne figurait donc pas (tout comme le PGE) parmi les documents consultables lors de l'enquête d'utilité publique qui s'est achevée en novembre 2012.

L'action de la CACG s'exerce par la relation «réalisation» du barrage, dont les valeurs positives correspondent à sa détermination à le construire.

\section{Le Collectif Testet}

Cet acteur regroupe le «Collectif pour la sauvegarde de la zone humide du Testet ${ }^{16}$ et les nombreuses organisations locales et nationales, telles que Lisle Environnement, Sauvegarde de l'environnement en pays rabastinois, Nature Midi-Pyrénées, Fédération nationale de l'environnement, ATTAC, Europe Écologie les Verts, etc., qui se sont mobilisées avec lui pour contester aussi bien la légalité que l'opportunité et le coût de ce barrage, ou bien critiquer (comme l'a fait le Parti communiste) la façon dont sa construction était menée. La Confédération paysanne est allée dans le même sens, ainsi que le journal en ligne Reporterre ${ }^{17}$ qui a suivi de près l'ensemble du projet. Le Collectif s'est doté d'une très forte expertise sur tous les aspects du dossier. Les institutions compétentes, à savoir le Conseil national de la protection de la nature (CNPN), le Comité départemental de la protection de la nature et de l'environnement (CDPNE) et l'Office national de l'eau et des milieux aquatiques (ONEMA), se sont prononcés dans le même sens que le Collectif. Il est avéré que l'État peut passer outre leurs avis, si bien que ces instances n'interviennent dans le jeu que par l'écho que le Collectif donne à leurs avis.

Le moyen d'action du Collectif est de faire valoir cette expertise auprès des autorités concernées, des tribunaux et de l'opinion publique. Les valeurs positives

\footnotetext{
${ }^{15}$ Comité de bassin Adour-Garonne: SDAGE du bassin Adour-Garonne 2010-2015, http://adour-garonne.eaufrance.fr/ upload/DOC/SDAGE/SDAGE_2010_2015.zip

${ }^{16}$ www.collectif-testet.org.

${ }^{17}$ www.reporterre.net.
}

de la relation « expertise » qu'il contrôle correspondent à une activité importante dans ce sens.

\section{Les zadistes}

Si le Collectif incarne la modalité légaliste - voire co-gestionnaire - de la contestation militante, les zadistes en incarnent la modalité activiste par l'occupation concrète $\mathrm{du}$ terrain. Nous regroupons sous ce terme toutes les personnes qui ont participé d'une façon ou d'une autre à l'occupation du secteur de construction du barrage, accompagnant ceux qui l'ont institué en zone à défendre ${ }^{18}$ à partir de l'automne 2013: participants aux rassemblements organisés sur place, hébergés occasionnellement ou ayant élu domicile sur la zone, venus de loin ou proches habitants apportant matériel et nourriture nécessaires à la vie quotidienne sur ce site isolé. Leurs motivations sont diverses, polarisées vers l'écologie ou vers la contestation des «grands projets inutiles imposés 》-défenseurs de la biodiversité, anticapitalistes, anarchistes, antispécistes, végans, etc. (Truong, 2014). De ce fait, ils ont des engagements (recherche d'un mode de vie dégagé de contraintes, égalitaire et autonome, pacifistes, rejet du barrage/des forces de l'ordre) et des comportements (engagement physique en s'attachant en haut des arbres ou en s'enterrant sur le passage des engins, clowneries, exactions, confrontations aux forces de l'ordre) extrêmement différenciés. Mais toute décomposition de la communauté des occupants en acteurs autonomes serait plus ou moins arbitraire et on ne considérera donc ici que l'effet émergent de ces comportements.

Cet acteur contrôle la relation « occupation », dont les valeurs positives correspondent à une forte entrave à la réalisation des travaux.

\section{D'autres acteurs}

Il existe d'autres acteurs parties prenantes au projet de barrage qui, pour diverses raisons, ne figurent pas parmi les acteurs du modèle.

L'agence de l'eau Adour-Garonne (AEAG)-Elle est un partenaire essentiel du projet, puisqu'elle le finance à $52 \%$. La politique de l'AEAG est de financer les ouvrages préconisés par les PGE et figurant au programme de mesures du SDAGE, ce qu'elle a décidé le 4 novembre 2013 concernant le barrage de Sivens, le ministre de l'Écologie Philippe Martin ayant levé les restrictions sur le financement de ce type d'ouvrage posées par sa prédécesseure Delphine Batho. En 2014, l'AEAG n'a donc plus matière à intervenir dans le déroulement du projet et il n'y a pas lieu de la considérer comme un acteur de notre modèle. À la suite du drame, la politique de l'AEAG a été de

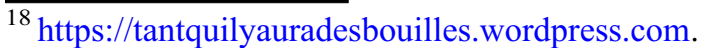


se tenir en retrait des conflits pour préserver sa légitimité dans le pilotage de l'élaboration du « projet de territoire ${ }^{19}$ » devant se substituer au PGE du Tescou.

L'Union européenne - Le barrage devait être financé à hauteur de $24 \%$ par l'UE, via le Fonds européen agricole de développement régional (FEADER). Mais dès novembre 2013, l'UE a demandé à Paris des explications sur ce projet, puis signifié en juillet 2014 la possibilité d'une procédure d'infraction à la directive cadre sur l'eau 2000/60/CE. L'État ayant ignoré ces avertissements, l'UE n'interviendrait comme acteur qu'à partir du déclenchement, le 26 novembre 2014, de cette procédure qui notifie l'impossibilité pour l'UE de financer ce barrage.

Le conseil régional-Même si le conseil régional Midi-Pyrénées a participé au financement d'études préliminaires, il s'est tenu à l'écart de ce dossier, n'étant pas décisionnaire.

Les riverains-Les «pro-barrage» sont regroupés avec le CG, les «anti-barrage» se répartissent entre le Collectif et les zadistes.

Les médias - Pour l'essentiel, les journaux nationaux ne se sont emparés du sujet qu'après la mort de Rémi Fraisse et ne sont donc pas intervenus dans le système d'action que nous considérons ici. Au niveau local, les principaux médias ne se sont pas engagés au point d'avoir un impact sur l'évolution du projet. Le principal journal de la région est La Dépêche du Midi dont le patron, Jean-Michel Baylet, est président du CG du Tarnet-Garonne (depuis 1985) et du Parti radical de gauche (depuis 1996). Ce journal a rendu compte factuellement des événements depuis les débuts du projet, mais sans analyser le projet sur le fond. Il en est de même de $F R 3$, qui a toutefois davantage donné la parole aux différentes parties. Le Tarn libre a suivi le projet de près, avec davantage d'analyse sur le fond.

\section{Le regroupement des acteurs}

Aucun des acteurs du modèle que nous avons identifiés ne constitue une entité homogène; chacun constitue en lui-même un système d'action, avec ses conflits internes et ses contradictions. Ce qui justifie les regroupements que nous avons opérés, ce sont les dépendances de chaque acteur du terrain et les effets de son comportement sur les autres. Plus précisément, considérons le réseau dont les nœuds sont les entités parties prenantes et comportant un arc, soit positif soit négatif, d'une entité A vers une

\footnotetext{
${ }^{19}$ Selon la circulaire du 4 juin 2015 de la ministre de l'Écologie donnant de nouvelles directives aux agences de l'eau, en suite au rapport d'Alain Richard (Richard, 2015) et répondant à la demande du président de la République lors de la conférence environnementale du 27 novembre 2014.
}

entité B selon la nature de l'impact du comportement de A sur B. La quantification du modèle montrera que les cinq acteurs de notre modèle correspondent aux regroupements qui maximisent les arcs positifs à l'intérieur de chaque groupe et les arcs négatifs entre les groupes.

\section{La quantification du modèle}

Notre modèle est donc constitué de cinq acteurs qui contrôlent chacun une relation (Tab. 1). La quantification des dépendances (enjeux et fonctions d'effet) de chaque acteur vis-à-vis des relations est établie à partir des données recueillies lors d'entretiens auprès de participants, en utilisant la grille donnée en annexe. Nous commentons ci-dessous les enjeux et fonctions d'effet présentés dans le tableau 2.

\section{L’État}

L'État ne place que deux points d'enjeux sur la relation qu'il contrôle, le «soutien», car il est suffisamment fort pour que son existence ne dépende qu'assez peu de son comportement au sein de ce projet. La courbe qui représente la fonction d'effet de la relation soutien sur l'État montre que ce qui convient le mieux à celui-ci est d'exercer son pouvoir, mais sans excès (le maximum est atteint pour une coopérativité de valeur 5 sur l'échelle $[-10,+10])$. Ne tenant pas compte des avis défavorables des commissions, l'État valorise assez peu l'expertise (1 point), et il se préoccupe davantage de l'occupation du site ( 2 points) dont il doit contrôler les possibles excès. La somme des enjeux de l'État n'est que de 5 points car il a une solidarité de 0,5 avec le $C G$ au titre de la libre administration des collectivités ${ }^{20}$. Ce soutien très fort au $\mathrm{CG}$ tient à la proximité du ministre Philippe Martin (entre autre président du CG du Gers, siège de la $\mathrm{CACG}$ ) avec les porteurs du projet et à l'engagement de la préfète en faveur du barrage. L'État ne dépend des relations « engagement » et «réalisation » que par l'intermédiaire de sa solidarité avec le CG.

\section{Le CG}

On trouvera dans l'analyse rétrospective réalisée par Foissac (2015) de précieux éclaircissements sur le comportement de Thierry Carcenac, le président du conseil général du Tarn. Le CG compte sur le soutien de l'État ( 2 points) dont les autorisations sont indispensables pour la réalisation du barrage. Fort de sa légitimité démocratique, le CG tient fortement à ce que le projet, voté par quarante-trois conseillers sur quarante-six, soit

\footnotetext{
${ }^{20}$ La satisfaction de l'État est donc constituée de la satisfaction qu'il retire des relations dont il dépend à laquelle s'ajoute la moitié de la satisfaction du CG.
} 
Tab. 2. Tableau de la dépendance, fonctions d'effet et enjeux, des acteurs (en colonne) vis-à-vis des relations (en ligne).

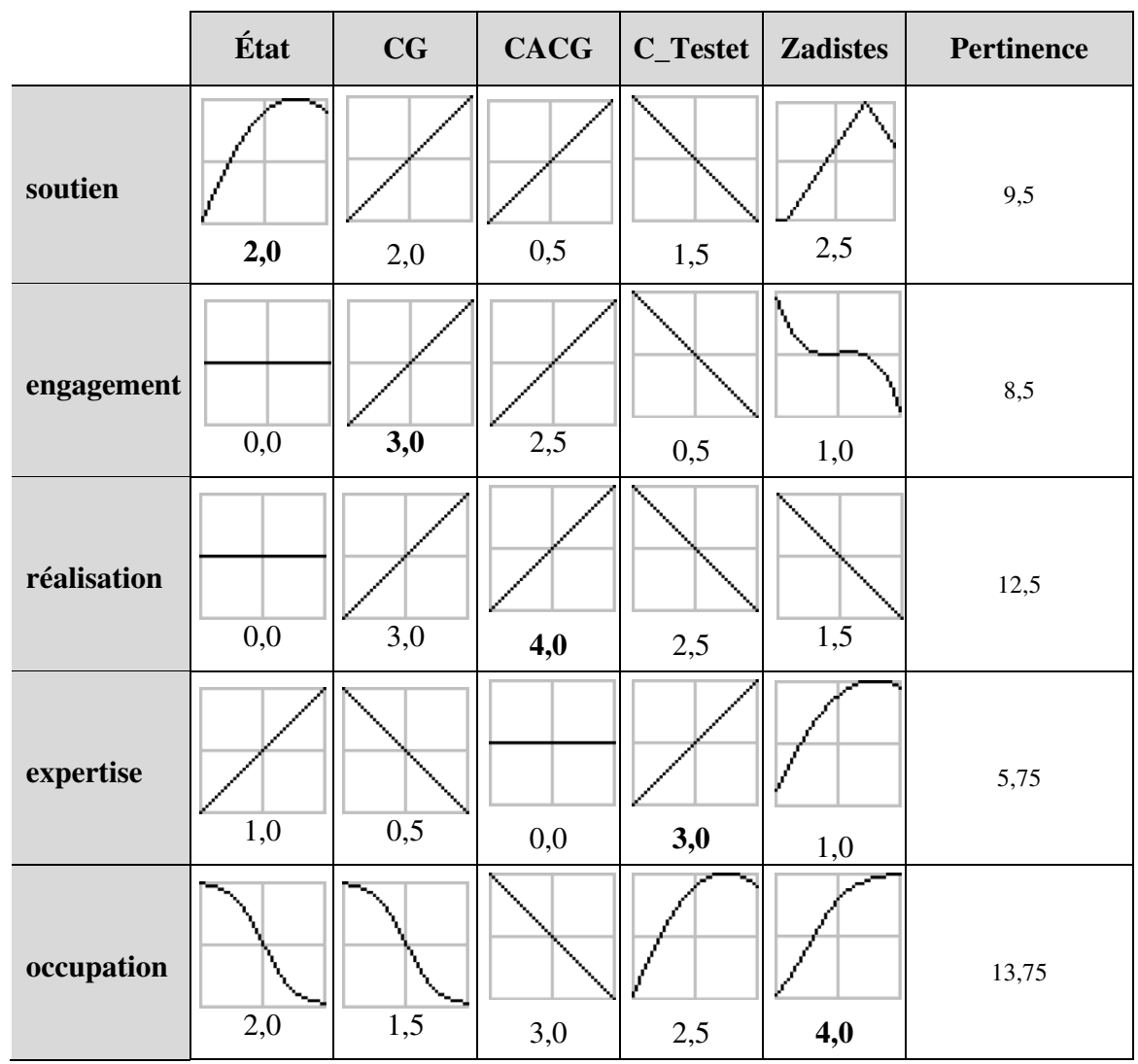

Sur la diagonale, la dépendance (l'enjeu est en gras) de l'acteur vis-à-vis de la relation qu'il contrôle.

Pour les fonctions d'effet, en abscisse le comportement de l'acteur qui contrôle la relation (de non coopératif à gauche vers coopératif à droite), en ordonnée la contribution à la satisfaction des objectifs de l'acteur dépendant.

La dernière colonne «pertinence» indique la somme des enjeux placés sur chaque relation en tenant compte des solidarités (chaque acteur a une solidarité de 1 envers lui-même et l'État a de plus une solidarité de 0,5 envers le CG).

Source : logiciel SocLab.

mené à bien (3 points), fût-ce au détriment de sa politique de préservation des zones humides ${ }^{21}$ et de sa charte de la citoyenneté (Foissac, 2015). Il tient tout autant à ce que la CACG mette tout en œuvre pour construire le barrage ( 3 points). Il tient peu compte de l'expertise $(0,5$ point: il ne donne pas suite aux demandes de rencontre de la part du Collectif) et la valorise négativement puisqu'elle remet en cause le projet. Quant à l'occupation de la zone, elle contrevient évidemment à ses objectifs et suscite à l'encontre des "peluts ${ }^{22}$ » une hostilité parfois violente de la part de l'Avet.

\footnotetext{
${ }^{21}$ En juin 2015, le lien sur le «pôle départemental des zones humides » (créé dès 2010) du site du conseil départemental du Tarn (http://zones-humides.tarn.fr) n'était plus actif.

${ }^{22}$ Mot occitan venant de "pelés », désignation péjorative et injurieuse de ceux qui ne «sont pas du pays».
}

\section{La CACG}

La CACG compte sur l'État pour pouvoir accéder au site $(0,5$ point $)$, mais avant tout sur l'engagement du $\mathrm{CG}$ (2,5 points). Société de droit privé dont le chiffre d'affaires a baissé de $10 \%$ entre 2012 et 2013, la réalisation de ce barrage est pour elle essentielle (4 points). Elle ne tient aucun compte de l'expertise et est très gênée par l'occupation du site ( 3 points).

\section{Le Collectif Testet}

Bien que les déclarations d'utilité publique et d'intérêt général et les autres autorisations aient été signées (octobre 2013), le Collectif compte encore sur l'Etat pour les référés qu'il dépose ( 1,5 point). Il n'espère plus beaucoup être entendu par le CG $(0,5$ point) et est très affecté par le déboisement qui détruit peu à peu la zone humide ( 2,5 points $)$. Sa propre expertise est essentielle pour le Collectif ( 3 points) car 
c'est ce qui fonde son existence et légitime son opposition au projet. Même si le Collectif tient à se démarquer des zadistes, notamment de leurs débordements, l'occupation du site reste son seul recours $(2,5$ points) face à «l'état de nécessité » instauré par le démarrage des travaux ${ }^{23}$.

\section{Les zadistes}

L'intervention des forces de l'ordre conforte l'action des zadistes en leur faisant de la publicité (2,5 points), jusqu'à un certain point au-delà duquel ils ne parviennent plus à empêcher la progression des travaux. Au-delà d'un certain niveau, les actions des milices dégradent réellement leurs conditions de vie quotidienne ${ }^{24}$ ( 1 point). Ils attachent moins d'importance à la construction du barrage (1,5 point) qu'à leur lutte emblématique contre l'État (2,5 points) (Bès et al., 2015). Même si les zadistes accordent un crédit limité à la stratégie légaliste du Collectif, son expertise justifie leur action et leur est indispensable (1 point), pour autant qu'elle n'occupe pas le devant de la scène. Pour eux, occuper la ZAD est essentiel (4 points) : c'est ce qui fonde leur identité. Certains sont conscients qu'un afflux excessif d'occupants nuit à l'instauration d'une culture commune, et donc compromet l'efficacité de l'action, d'autant que les ressources du site sont limitées. D'autres, moins politisés ou moins expérimentés, valorisent l'affrontement en lui-même et, par exemple, n'hésitent pas à se confronter aux forces de l'ordre dont la présence est considérée comme une provocation. La fonction d'effet de l'occupation sur les zadistes correspond à la résultante de ces deux tendances.

\section{Analyse du modèle}

La mort d'un opposant au projet lors d'affrontements avec les forces antiémeutes est un événement dont la possibilité s'accroît avec l'importance du soutien de l'État (soutien à 10) et la vigueur de l'occupation de la

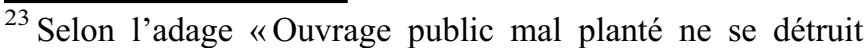
pas », le barrage restera même si le tribunal administratif le déclare illégal. C'est le cas à proximité du barrage de Fourogue, déclaré illégal en 2001, construit et exploité par la CACG depuis 1998 et faisant l'objet d'une nouvelle enquête d'utilité publique en octobre 2016 pour permettre le financement de réparations alors qu'il est censé ne pas exister. Les travaux ont commencé à Sivens sans accord de financement par l'UE, refusé en novembre 2014.

${ }^{24} \mathrm{Il}$ semblerait que, sur le terrain, les zadistes aient sous-estimé l'impact sur la viabilité de leur microsociété des agressions des milices contre lesquelles il leur était très difficile de se prémunir, alors que le comportement des forces de l'ordre reste relativement cadré par les règles d'engagement.
}

zone (occupation à 10). Ce sont donc les deux relations que nous considérerons avec le plus d'attention.

\section{Analyse structurelle}

L'analyse structurelle d'un modèle permet de mettre en évidence certaines de ses propriétés intrinsèques et le jeu des possibles. Le tableau 3 montre les configurations les plus satisfaisantes pour chacun des acteurs, et celles le plus et le moins satisfaisantes globalement (i.e. en faisant la somme de la satisfaction des acteurs). Il apparaît que chacun aurait la possibilité de réaliser ses objectifs (satisfactions entre 90,4 et 100)... pour autant que les autres y consentent.

En termes de conflit, la comparaison des colonnes de ce tableau ${ }^{25}$ montre que le conflit principal est entre l'État, le CG et la CACG d'une part et le Collectif et les zadistes d'autre part (les satisfactions maximales des uns correspondent à des satisfactions négatives des autres, les maxima et minima globaux distinguent clairement les deux groupes); que les intérêts de l'État sont ceux les plus convergents avec l'intérêt général (la configuration de son maximum de satisfaction procure une satisfaction globale de 199,8, plus élevée que celle des autres acteurs)-rien de surprenant à cela, qu'il en soit autrement remettrait en cause la validité du modèle; que le Collectif est plus particulièrement en conflit avec le CG et les zadistes avec la CACG. Ce dernier résultat attire l'attention sur un fait significatif: l'opposition directe sur le terrain se joue entre les zadistes et la $\mathrm{CACG}$, et celle relative à la légalité et l'opportunité du projet entre le Collectif et le CG.

Quelle est l'amplitude du pouvoir que chaque acteur peut exercer, c'est-à-dire sa capacité à contribuer à la réalisation des objectifs des autres et ainsi influencer leurs comportements?

La pertinence des relations (Tab.2) pourrait faire croire que les zadistes et la CACG sont les acteurs les plus puissants puisque c'est sur les relations qu'ils contrôlent que se portent le plus d'enjeux $(13,75$ et 12,5 points respectivement). Il n'en est rien. Le tableau 4 montre, pour chaque acteur, le comportement que celui-ci adopte et le pouvoir qu'il exerce lorsqu'il minimise ou maximise son pouvoir, et en déduit l'amplitude de ce pouvoir ${ }^{26}$. C'est donc le CG qui dispose de la plus grande marge de manœuvre au regard de l'influence qu'il exerce sur les autres - après tout c'est

\footnotetext{
${ }^{25}$ Ces résultats sont confirmés par les configurations correspondant aux minima de satisfaction.

${ }^{26}$ Le comportement qui maximise ou minimise le pouvoir qu'exerce un acteur est celui qu'il adopte dans la configuration du maximum ou du minimum de satisfaction globale (première et dernière colonne du tableau 3 ).
} 
Tab. 3. Maxima de satisfaction des acteurs.

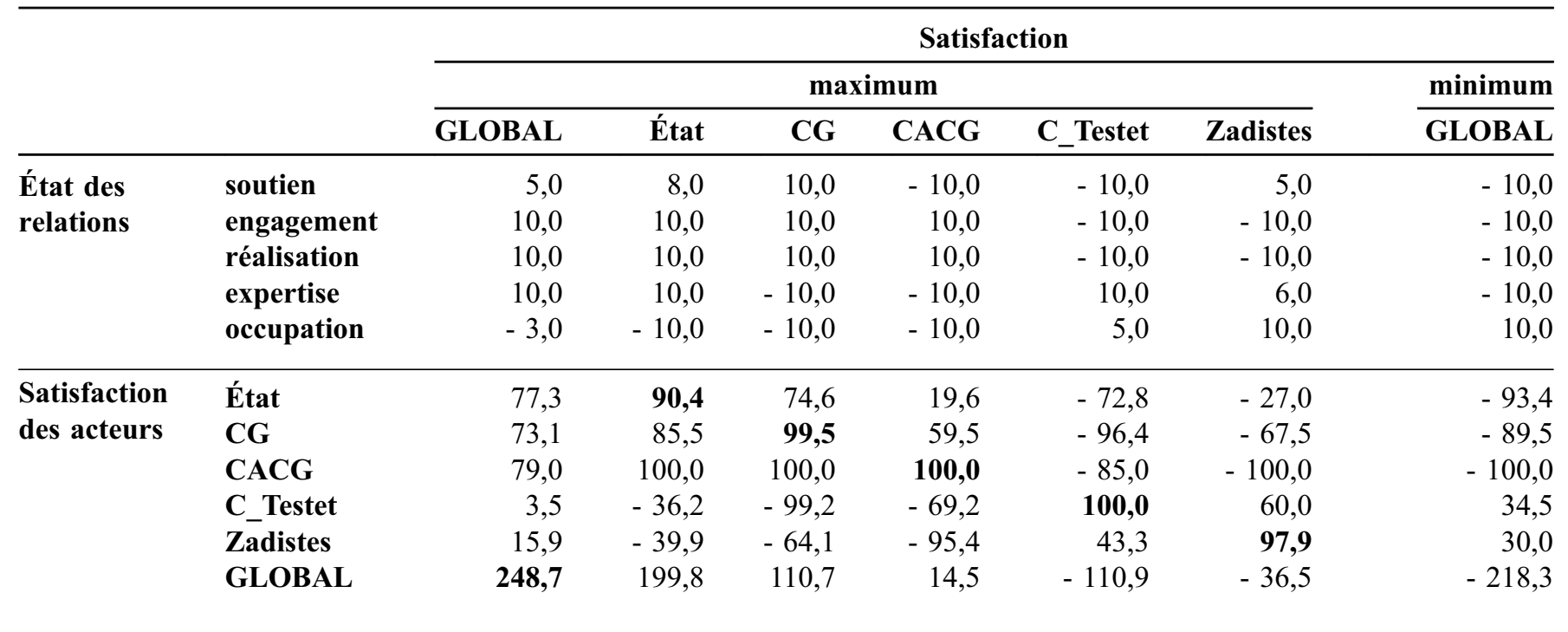

En colonne, les configurations correspondant aux extrema de satisfaction (par exemple, la $2^{\mathrm{e}}$ colonne correspond à la configuration qui procure le maximum de satisfaction à l'État, soit 90,4).

En ligne, l'état de chaque relation dans la configuration considérée et la satisfaction qui en résulte pour chaque acteur (en gras, valeur du maximum de la satisfaction de l'acteur).

Source: logiciel SocLab.

le maître d'ouvrage du projet-, influence équivalente au double de celle des zadistes.

Le «mieux» (la configuration du maximum global) serait que l'État soutienne modérément (comportement de 5) et que les zadistes occupent peu (comportement de - 3), éloignant ainsi la possibilité d'un accident. Mais les perdants sont-ils susceptibles de s'y résoudre sans faire d'histoires?

\section{Résultats de simulation}

L'algorithme de simulation de SocLab apporte une réponse à cette question en calculant des configurations dans lesquelles il est plausible que le jeu des acteurs se régule. Les simulations sont répétées car l'algorithme comporte une part d'aléa (quand un acteur ne sait quel comportement adopter, il le choisit au hasard). Les résultats de simulation, dont on trouvera les détails dans l'article de Sibertin-Blanc (2016), se prêtent donc à des analyses statistiques (Villa-Vialaneix et al., 2014); on ne s'intéresse ici qu'à la moyenne et la dispersion des variables. La déviation de l'état d'une relation est un indicateur de la marge de manœuvre, ou de l'indécision, de l'acteur qui contrôle cette relation. Le tableau 5 montre ces résultats.

En ce qui concerne l'état des relations, l'État soutient (comportement de 10) et les zadistes occupent (compor- tement de 10) au maximum de leurs possibilités; les circonstances de la survenue d'un accident sont donc réunies. De plus, la déviation est nulle, toutes les simulations conduisent exactement à la même configuration de blocage : le jeu est surdéterminé, aucun acteur ne voit comment il pourrait se comporter autrement.

En ce qui concerne la satisfaction des acteurs, le CG apparait comme le gagnant et le Collectif et les zadistes comme les perdants, les autres acteurs étant dans une situation intermédiaire. Ces résultats correspondent à ce qui aurait dû se passer si le jeu n'avait pas été dramatiquement interrompu par la mort d'un opposant : le bétonnage de la digue devait commencer le 27 octobre. Hormis l'Etat, les comportements adoptés par les acteurs correspondent à un équilibre de Nash: chacun adopte le comportement qui lui convient le mieux, ne comptant pas sur les autres pour réaliser ses objectifs. Cette régulation est d'autant plus stable qu'elle est conforme à l'intérêt de la majorité - CG, la CACG et le Collectif $(100 \%$ de leur pouvoir) - quoiqu'opposée à celui des zadistes. Ces résultats sont très robustes: les simulations de cent modèles obtenus en choisissant pour chaque enjeu une valeur aléatoire dans l'intervalle de plus ou moins 1,5 point autour de sa valeur de référence (tout en maintenant à 10 le total des enjeux de chaque acteur), produisent cette même configuration avec une déviation de 0,03 en moyenne pour chaque relation. 
Tab. 4. L'amplitude du pouvoir que chaque acteur est en mesure d'exercer.

\begin{tabular}{|c|c|c|c|c|c|c|c|c|c|c|}
\hline & \multicolumn{2}{|c|}{ État } & \multicolumn{2}{|c|}{$\mathbf{C G}$} & \multicolumn{2}{|c|}{ CACG } & \multicolumn{2}{|c|}{ C_Testet } & \multicolumn{2}{|c|}{ Zadistes } \\
\hline maximum & 5 & 53 & 10 & 61 & 10 & 45 & 10 & 41 & -3 & 49 \\
\hline amplitude & & 112 & & 122 & & 90 & & 82 & & 61 \\
\hline
\end{tabular}

Source : logiciel SocLab.

Tab. 5. Résultats de 100 simulations qui donnent toutes la configuration de blocage.

\begin{tabular}{|c|c|c|c|c|c|c|c|}
\hline & \multicolumn{2}{|c|}{ état des relations } & & \multicolumn{2}{|c|}{ satisfaction des acteurs } & \multicolumn{2}{|c|}{ pouvoir des acteurs } \\
\hline & moyenne & déviation & & valeur & proportion & valeur & proportion \\
\hline engagement & 10,0 & 0,0 & $\mathbf{C G}$ & 61 & $80 \%$ & 61 & $100 \%$ \\
\hline réalisation & 10,0 & 0,0 & CACG & 40 & $70 \%$ & 45 & $100 \%$ \\
\hline occupation & & & GLOBAL & 172 & $84 \% *$ & 172 & $84 \%$ \\
\hline
\end{tabular}

* L'amplitude de la satisfaction globale est inférieure à la somme de l'amplitude des satisfactions des acteurs car leurs maxima et leurs minima ne coïncident pas.

Source : logiciel SocLab.

\section{Modèles pour une modération du conflit État-zadistes}

Quelles autres représentations et stratégies des acteurs modifieraient la structure du jeu dans le sens d'une modération du conflit entre l'État et les zadistes?

Du côté de l'État, on pourrait envisager que sa mobilisation des forces de l'ordre reste proportionnée et que sa solidarité avec le CG au titre de la libre administration des collectivités n'excède pas son attention à l'expertise.

Du côté du principal intéressé, le CG maître d'ouvrage, on pourrait s'attendre à ce qu'il donne moins de gages aux milieux agricoles et qu'il valorise positivement l'expertise du Collectif.

Du côté de la CACG et du Collectif, leur représentation est l'expression de leur raison d'être et on ne voit pas bien comment ils pourraient en changer.

Pour les zadistes enfin, mieux organisés ils auraient pu se coordonner pour contrôler davantage les excès de certains d'entre eux.

Ces autres représentations et stratégies possibles des acteurs nous conduisent à considérer un deuxième modèle dans lequel :
- la distribution des enjeux de l'État et du CG est modifiée (Tab. 6), la solidarité de l'État avec le CG étant ramenée à 0,3 ;

- les fonctions d'effet sont inchangées par rapport au tableau 2, à l'exception de la fonction de la relation expertise sur le CG dont la pente devient croissante;

- la valeur de l'état de la relation occupation est bornée à 7 .

Dans les résultats de simulation de ce modèle, la déviation de la relation "soutien» est de 3,3, ce qui conduit à regarder la dispersion des résultats. Il s'avère (Tab. 7) qu'un tiers des simulations donnent exactement la même configuration de blocage que précédemment (Tab. 5), hormis la limitation de l'occupation à 7, alors que les deux autres tiers conduisent à des configurations de modération dans lesquelles l'État ne soutient qu'à hauteur de 3,4 et les zadistes occupent à hauteur de 5,4. Il y a donc deux chances sur trois que les circonstances de la survenue d'un accident ne soient pas réunies. Dans les configurations de modération, si le CG, la CACG et le Collectif adoptent quasiment le même comportement que dans le premier modèle, le changement escompté, une modération du soutien de l'État et de l'occupation par les zadistes, est bien obtenue. Il en résulte une amélioration de $10 \%$ de la satisfaction globale, au bénéfice de l'État, du Collectif et des zadistes (gain d'environ 15 points de 
Tab. 6. Tableau des enjeux des acteurs vis-à-vis des relations dans le modèle 2.

\begin{tabular}{lrrrrrr}
\hline & État & CG & CACG & C_Testet & Zadistes & Pertinence \\
\hline soutien & $\mathbf{2 , 0}$ & 2,0 & 0,5 & 1,5 & 2,5 & 9,1 \\
engagement & 0,0 & $\mathbf{2 , 0}$ & 2,5 & 0,5 & 1,0 & 6,6 \\
réalisation & 0,0 & 2,5 & $\mathbf{4 , 0}$ & 2,5 & 1,5 & 11,25 \\
expertise & 3,0 & 2,0 & 0,0 & $\mathbf{3 , 0}$ & 1,0 & 9,6 \\
occupation & 2,0 & 1,5 & 3,0 & 2,5 & $\mathbf{4 , 0}$ & 13,45 \\
\hline
\end{tabular}

En gras, l'enjeu que l'acteur place sur la relation qu'il contrôle.

Source: logiciel SocLab.

Tab. 7. Résultats de 100 simulations du modèle 2 .

\begin{tabular}{|c|c|c|c|c|c|c|c|c|c|}
\hline & \multicolumn{4}{|c|}{ état des relations } & & \multicolumn{2}{|c|}{ satisfaction des acteurs } & \multicolumn{2}{|c|}{ pouvoir des acteurs } \\
\hline & \multicolumn{2}{|c|}{ blocage } & \multicolumn{2}{|c|}{ modération } & \multirow[b]{2}{*}{ État } & moyenne & \multirow{2}{*}{$\begin{array}{r}\text { proportion } \\
77 \%\end{array}$} & \multirow{2}{*}{$\begin{array}{r}\text { moyenne } \\
46\end{array}$} & \multirow{2}{*}{$\frac{\text { proportion }}{99 \%}$} \\
\hline & moyenne & déviation & moyenne & déviation & & 50 & & & \\
\hline soutien & 10,0 & 0,0 & 3,4 & 0,6 & CG & 58 & $79 \%$ & 36 & $99 \%$ \\
\hline engagement & 10,0 & 0,0 & 9,5 & 0,8 & CACG & 48 & $74 \%$ & 32 & $99 \%$ \\
\hline réalisation & 10,0 & 0,0 & 9,7 & 0,7 & C_Testet & 20 & $60 \%$ & 94 & $100 \%$ \\
\hline expertise & 10,0 & 0,0 & 9,9 & 0,2 & Zádistes & 45 & $73 \%$ & 13 & $40 \%$ \\
\hline occupation & 7,0 & 0,0 & 5,4 & 1,2 & GLOBAL & 221 & $94 \%$ & 221 & $94 \%$ \\
\hline
\end{tabular}

Les résultats concernant les relations distinguent les simulations qui conduisent au blocage et celles qui conduisent à la modération.

Les résultats concernant les acteurs portent sur les seules configurations modérées.

Source: logiciel SocLab.

satisfaction chacun). L'issue du jeu est beaucoup plus équilibrée : le Collectif n'est plus ridiculisé, l'État exerce pleinement son pouvoir et il n'est pas sûr que le barrage soit construit.

Quels sont les déterminants de cette évolution des comportements? Ce n'est pas le changement de représentation du CG, pourtant le premier intéressé et le plus puissant des acteurs (Tab. 4): on obtient la configuration de blocage même s'il accorde davantage d'enjeux à l'expertise qu'à la réalisation du barrage. Il en est de même du plafonnement à 7 de l'occupation par les zadistes, qui, si rien d'autre n'est modifié dans le modèle, a pour seul effet de limiter l'état de cette relation. C'est donc le changement de représentation de l'État qui ouvre une alternative au blocage.

Pour que la configuration de blocage soit franchement écartée, il faut que l'État, simple régulateur, abandonnant sa solidarité avec le CG, dissocie et accorde une égale importance aux aspects «expertise-autorisation » et «occupation-maintien de l'ordre». C'est ce que fait le modèle 3, dont la structure est donnée dans SibertinBlanc (2016) et dont les résultats de simulation sont présentés dans le tableau 8 . Ces résultats sont robustes: ils ne sont pas sensibles à une variation de \pm 1 des enjeux des acteurs.

Quelques propriétés se retrouvent dans les résultats de simulation de chacun de ces trois modèles; elles sont le reflet de caractéristiques (de notre modélisation) du jeu d'acteur qui méritent d'être relevées :

- La valeur des relations engagement, réalisation et expertise est proche de leur valeur maximale avec une faible déviation, alors que la déviation des relations contrôlées par l'État et les zadistes est significative. Cela tient à la focalisation des modèles sur le conflit entre l'État et les zadistes ; leur représentation de ce qui est en jeu est plus complexe et leur laisse une plus grande marge de manœuvre, alors que les trois autres acteurs figurent essentiellement comme auxiliaires de ce conflit.

- Le Collectif est l'acteur qui obtient toujours la moindre satisfaction, bien que sa contribution au collectif (94 points dans le modèle 2 ) puisse être très importante; l'opposition légaliste est un rôle ingrat.

- Par contre, les zadistes exercent toujours un pouvoir très faible; ils ont leur propre façon de jouer le jeu, qui ne s'accorde pas avec celle des autres acteurs. 
Tab. 8. Résultats de simulation du modèle 3 qui écarte la possibilité d'un accident dramatique.

\begin{tabular}{|c|c|c|c|c|c|c|c|}
\hline & \multicolumn{2}{|c|}{ état des relations } & & \multicolumn{2}{|c|}{ satisfaction des acteurs } & \multicolumn{2}{|c|}{ pouvoir des acteurs } \\
\hline & moyenne & déviation & & valeur & proportion & valeur & proportion \\
\hline autorisations & $-4,1$ & 2,06 & État & 43,4 & $68,5 \%$ & 61,4 & $99,6 \%$ \\
\hline engagement & 9,1 & 1,12 & CG & 37,8 & $68,5 \%$ & 24,5 & $98,5 \%$ \\
\hline réalisation & 9,8 & 0,36 & CACG & 43,3 & $71,8 \%$ & 24,4 & $99,0 \%$ \\
\hline expertise & 9,9 & 0,13 & C_Testet & 22,8 & $61,9 \%$ & 78,3 & $99,6 \%$ \\
\hline occupation & 6,3 & 2,1 & Zädistes & 41,0 & $73,2 \%$ & 0,1 & $27,2 \%$ \\
\hline maintienOrdre & 5,1 & 1,2 & GLOBAL & 188,4 & $87,3 \%$ & 188 & $87,0 \%$ \\
\hline
\end{tabular}

L'État contrôle les relations «autorisation» et «maintien de l'ordre».

Source: logiciel SocLab.

\section{Conclusion}

Dans leur rapport remis le 27 octobre 2014, et donc rédigé avant la survenue du drame, à la ministre de l'Écologie, Forray et Rathouis (2014) souhaitaient que «Sivens soit considéré comme un tournant dans la gestion de l'eau en Adour-Garonne, dernier projet d'une époque, première étape d'une évolution majeure». Il semble qu'il doive bien en être ainsi avec, notamment, la circulaire du 4 juin 2015 relative au financement par les agences de l'eau des retenues de substitution et la loi du 8 août 2016 pour la reconquête de la biodiversité, de la nature et des paysages suites au rapport établi par Alain Richard (Richard, 2015), ou la modification de la nature et des modalités d'engagement des munitions utilisables dans les opérations de maintien de l'ordre suite à l'élaboration de plusieurs rapports (Renault et al., 2014 ; Baudet et Miramon, 2014).

Il est déplorable qu'il ait fallu une tragédie pour que soient révélées les défaillances du projet de barrage de Sivens, tant du point de vue de sa gestion que de son objet (la gestion de l'eau), et que soit stoppée sa mise en œuvre. Par rapport à des projets complexes aux multiples enjeux enchevêtrés tels que l'aéroport de Notre-Dame-desLandes, le Center Parcs de Roybon ou la ferme des mille vaches, les enjeux de ce projet étaient relativement modestes : $8 \mathrm{M} €, 12$ à 17 ha de zone humide et la culture $\mathrm{du}$ maïs semence dans une vingtaine d'exploitations agricoles. Dans la mesure où ses attendus, enjeux et dysfonctionnements sont relativement faciles à appréhender, le projet de barrage de Sivens est donc un paradigme des "grands projets inutiles imposés » qui donnent lieu à de graves conflits entre les systèmes administratif et politique français et des mouvements citoyens porteurs d'une conception du bien commun qui se veut durable, riche et efficace des points de vue environnemental, social et économique (Foissac, 2015).

Au-delà des évolutions législatives et réglementaires évoquées ci-dessus, la prise de conscience des consé- quences désastreuses de tels hiatus semble encourager le recours à la Commission nationale du débat public en ce qui concerne la légitimité démocratique de ces projets. Elle met également en évidence la nécessité de démarches de concertation participative (Barbier et Larrue, 2011) pour l'élaboration d'un projet qui soit partagé et assumé par les acteurs d'un territoire, et donc l'importance, en support à ces processus, des méthodes et outils à base de modélisation intégrative et de simulation tels que Wat-A-Game (Abrami et al., 2012) ou Maelia (Thérond et al., 2014). Utilisées comme outils d'aide à la négociation pour la gestion des systèmes socioécologiques (Barreteau, 2003; Collectif ComMod, 2005 ; Adreit et al., 2011), la modélisation permet d'objectiver les positions et comportements des parties prenantes et la simulation de tirer les conséquences des choix de ces dernières.

Un modèle SocLab tel que celui présenté ici se situe à un autre niveau, puisqu'il ne porte pas sur l'objet du projet mais sur la dimension sociale de sa conception et de sa gestion; ce modèle relève de l'aide au diagnostic dans la mesure où il est problématisé autour d'une question qu'il vise à éclairer (en l'occurrence le conflit Etat-zadistes). Compte tenu de la question étudiée, les modèles présentés ici se situent à un niveau méso, intermédiaire entre le niveau micro qui examinerait les interactions entre les acteurs concrets du terrain et le niveau macro pour lequel le projet de barrage de Sivens ne constituerait qu'un épisode parmi d'autres.

Le méta-modèle SocLab des systèmes d'action organisée fournit en premier lieu un guide pour l'acquisition (voir annexe) et la représentation (Tab. 1 et 2) de connaissances relatives au système d'action que l'on considère. Le caractère quantitatif de cette représentation permet de mettre en évidence des propriétés qui, pour être la conséquence directe de ce que l'on a mis dans le modèle, ne sont pas pour autant toutes triviales et dont la robustesse peut être évaluée par des analyses de sensibilité. 
Enfin, SocLab offre la possibilité de tester toutes sortes d'hypothèses en observant les conséquences de modifications apportées au modèle. Chacun de ces tests constitue une expérimentation capable d'étendre la connaissance du modèle et d'en approfondir la compréhension. C'est ainsi que nous avons pu dévoiler des éléments de la structure du système d'action étudié susceptibles d'en être les déterminants essentiels.

\section{Références}

Abrami G., Ferrand N., Morardet S., Murgue C., Popova A., De Fooij H., Farolfi S., Du Toit D., Aquae-Gaudi W., 2012. Wat-A-Game, a toolkit for building role-playing games about integrated water management, in Seppelt R., Voinov A.A., Lange S., Bankamp D. (Eds), Proceedings of the $6^{\text {th }}$ International Congress on Environmental Modelling and Software, Leipzig, iEMSs, 1912-1919.

Adreit F., Roggero P., Sibertin-Blanc C., Vautier C., 2011. Using SocLab for a rigorous assessment of the social feasibility of agricultural policies, International Journal of Agricultural and Environmental Information Systems, 2, 2, 1-20, doi: 10.4018/jaeis.2011070101.

Adreit F., Chapron P., El Gemayel J., Roggero P., Sandri S., Sibertin-Blanc C., 2014. Une formalisation de la sociologie de l'action organisée: métamodèle, simulation et étude analytique, in Phan D. (Ed.), Ontologies et modélisation par SMA en SHS, Paris, Hermès, 374-398.

Axelrod R., 1997. Advancing the art of simulation in the social sciences, in Conte R., Hegselmann R., Terna P. (Eds), Simulating social phenomena. Lecture notes in economics and mathematical systems, Berlin, Heidelberg, SpringerVerlag, 21-40.

Barbier R., Larrue C., 2011. Démocratie environnementale et territoires: un bilan d'étape, Participations, 1, 1, 67-104, doi: $10.3917 /$ parti.001.0067.

Barreteau O., 2003. The joint use of role-playing games and models regarding negotiation processes: characterization of associations, Journal of Artificial Societies and Social Simulation, 6, 2, http://jasss.soc.surrey.ac.uk/6/2/3.html.

Baudet M., Miramon G., 2014. Rapport relatif à l'emploi des munitions en opérations de maintien de l'ordre. Rapport, Paris, ministère de l'Intérieur, www.ladocumentationfran caise.fr/var/storage/rapports-publics/144000676.pdf.

Bès M-P., Blot F., Ducournau P., 2015. Sivens: quand le dialogue devient impossible. Chronique d'un drame annoncé, Justice spatiale, 8, https://www.jssj.org/article/ sivens-quand-le-dialogue-devient-impossible-chroniquedun-drame-annonce.

CACG (compagnie d'aménagement des coteaux de Gascogne), 2001. Confortement de la ressource en eau sur le bassin du Tescou. Rapport final, Tarbes, conseil général du Tarn-et-Garonne, www.collectif-testet.org/79+telechargerle-rapport-cacg-2001.html.
Callon M., 1986. The sociology of an actor-network, in Callon M., Law J., Rip A. (Eds), Mapping the dynamics of science and technology, London, Macmillan, 19-34.

Collectif, 2014. Sivens sans retenue : feuilles d'automne, Paris, Éditions La lenteur.

Collectif, 2015. R COMM..., Villefranche-de-Rouergue, Éditions QuandMême!, https://cras31.info/IMG/pdf/r_comme._collec tif.pdf.

Collectif ComMod, 2005. La modélisation comme outil d'accompagnement, Natures Sciences Sociétés, 13, 2, 165168, doi: 10.1051/nss:2005023.

Cour des comptes, 2015. Le rapport public annuel 2015, Tome 1. Les agences de l'eau et la politique de l'eau: une cohérence à retrouver. Rapport, Paris, Cour des comptes, https://www.ccomptes.fr/sites/default/files/EzPublish/112RPA2015-agences-et-politique-de-1-eau.pdf.

Crozier M., 1963. Le phénomène bureaucratique. Essai sur les tendances bureaucratiques des systèmes d'organisation modernes et sur leurs relations avec le système social et culturel, Paris, Le Seuil.

Crozier M., Friedberg E., 1977. L'acteur et le système: Les contraintes de l'action collective, Paris, Le Seuil.

El Gemayel J., Chapron P., Adreit F., Sibertin-Blanc C., 2011. La coopération des acteurs sociaux. Un algorithme de simulation pour la négociation de leurs comportements, Revue d'Intelligence Artificielle, 25, 1, 43-67, doi: 10.3166/ ria.25.43-67.

Foissac R., 2015. Sivens, pour comprendre, Albi, Éditions Un Autre Reg'Art.

Forray N., Rathouis P., 2014. Expertise du projet de barrage de Sivens (Tarn). Rapport, conseil général de l'Environnement et du Développement durable, ministère de l'Écologie, du Développement durable et de l'Énergie, www.ladocumenta tionfrancaise.fr/var/storage/rapports-publics/144000641. pdf.

Forray N., Roche P-A., 2015. Mission pour un projet de territoire du bassin du Tescou (Midi-Pyrénées). Rapport, conseil général de l'Environnement et du Développement durable, ministère de l'Écologie, du Développement durable et de l'Énergie, www.ladocumentationfrancaise. $\mathrm{fr} / \mathrm{var} /$ storage/rapports-publics/154000052.pdf.

GéoDiag, Ecogéa, 2007. Recensement des cours d'eau et des milieux aquatiques à «caractère patrimonial » sur le bassin Adour-Garonne-Cours d'eau remarquables. Rapport final, Pau, Pins-Justaret, Agence de l'Eau Adour-Garonne, Toulouse, novembre 2007.

Gilbert N., 2004. Quality, quantity and the third way, in Holland J., Campbell J. R. (Eds), Methods in development research: combining qualitative and quantitative approaches, London, ITDG Publications, 141-148.

Grossetti M., 2004. Sociologie de l'imprévisible. Dynamiques de l'activité et des formes sociales, Paris, Presses Universitaires de France.

Lefetey B., 2015. Sivens, un barrage contre la démocratie, Paris, Éditions Les petits matins. 
Marcaillou L., 2014. Le bassin Adour-Garonne manque d'eau, Les Échos, https://www.lesechos.fr/28/10/2014/LesEchos/ 21802-096-ECH_le-bassin-adour-garonne-manque-d-eau. htm.

Milanesi J., Sébastien L., Grisoni A., Pelenc J., 2018. Résister aux grands projets inutiles et imposés, de Notre-Dame-desLandes à Bure, Paris, Éditions Textuel.

Renault P., Betton M., Anin R., 2014. Rapport d'enquête administrative relative a la conduite des opérations de maintien de l'ordre dans le cadre du projet de barrage de Sivens (Tarn). Rapport, Paris, Inspection générale de la gendarmerie nationale, ministère de l'Intérieur, www. ladocumentationfrancaise.fr/var/storage/rapports-publics/ 144000731.pdf.

Richard A., 2015. Démocratie environnementale : débattre et décider. Rapport, Conseil national de la transition écologique sur la démocratisation du dialogue environnemental, https://www.ladocumentationfrancaise.fr/var/sto rage/rapports-publics/154000364.pdf.

Scop Sagne, 2010. Retenue de Sivens. Caractérisation des zones humides. Rapport, conseil général du Tarn, www. collectif-testet.org/uploaded/Etude-impact-EP-2012/Anne xes-EI/annexe-4-rapportzh-na-1.pdf.

Sibertin-Blanc C., 2016. Interplay of actors about the construction of a dam, CoMSES Computational Model Library, https://www.openabm.org/model/5307.

Sibertin-Blanc C., El Gemayel J., 2013. Boundedly rational agents playing the social actors game-How to reach cooperation, in O'Conner L. (Ed.), Proceedings of IEEE/ WIC/ACM International Joint Conference on Web Intelligence and Intelligent Agent Technology, Atlanta, IEEE, 375-382 (version pré-print disponible sur Hal, https://hal. archives-ouvertes.fr/hal-01147310).
Sibertin-Blanc C., Roggero P., Adreit F., Baldet B., Chapron P., El Gemayel J., Mailliard M., Sandri S., 2013. SocLab: a framework for the modeling, simulation and analysis of power in social organizations, Journal of Artificial Societies and Social Simulation, 16, 4, 8, http://jasss.soc.surrey.ac. uk/16/4/8.html.

Simon H.A., 1982. Models of bounded rationality. Volume 1: Economic analysis and public policy. Volume 2: Behavioral economics and business organization, Cambridge, MA, MIT Press.

Souchay G., Laimé M., 2015. Sivens, le barrage de trop, Paris, Seuil-Reporterre.

Subra P., 2014. Géopolitique de l'aménagement du territoire, Paris, Armand Colin.

Thérond O., Sibertin-Blanc C., Lardy R., Gaudou B., Balestrat M., Hong H., Louail T., Nguyen V.B., Panzoli D., SanchezPerez J-M., Sauvage S., Taillandier P., Vavasseur M., Mazzega P., 2014. Integrated modelling of social-ecological systems: the MAELIA high-resolution multi-agent platform to deal with water scarcity problems, in Ames D. P., Quinn N.W.T. (Eds), Proceedings of the $7^{\text {th }}$ International Congress on Environmental Modelling and Software, San Diego, iEMSs (version pré-print disponible sur Hal, https:// hal.archives-ouvertes.fr/hal-01360865).

Truong N., 2014. La résistance des renoncules, Le Monde 4/11/2014, https://www.lemonde.fr/idees/article/2014/11/ 04/la-resistance-des-renoncules_4517837_3232.html.

Villa-Vialaneix N., Sibertin-Blanc C., Roggero P., 2014. Statistical exploratory analysis of agent-based simulations in a social context: using self-organizing maps to define a typology of behaviors, Case Studies in Business, Industry and Government Statistics, 5, 2, 132-149 (version pré-print disponible sur Hal, https://hal.archives-ouvertes.fr/hal00940788/document). 


\section{Annexe. Formulaire de recueil de données}

Ce formulaire est utilisé pour recueillir auprès des acteurs de terrain les données permettant de déterminer les acteurs du modèle et de quantifier le modèle, i.e. de remplir les colonnes du tableau 2. On commence par présenter à l'interlocuteur l'architecture du modèle à l'aide de la figure 1 et par lui expliquer l'interprétation des fonctions d'effet, afin qu'il sache comment ses dires seront utilisés pour remplir une colonne du tableau 2. La délimitation des acteurs du modèle s'effectue progressivement, au fil des entretiens.

\begin{tabular}{|c|c|c|c|c|}
\hline & \multirow{2}{*}{$\begin{array}{l}\text { Personne interrogée }: \ldots \ldots \ldots \\
\text { Acteur du modèle }: \ldots \ldots \ldots \ldots\end{array}$} & \multicolumn{3}{|c|}{ Ressources } \\
\hline & & R1 & $\mathbf{R} 2$ & -- \\
\hline \multicolumn{5}{|c|}{ Détermination des enjeux } \\
\hline 1. & $\begin{array}{l}\text { Quelles sont les ressources dont vous avez besoin pour } \\
\text { parvenir à vos fins? Qu'est-ce qui importe pour vous? }\end{array}$ & & & \\
\hline 2. & $\begin{array}{l}\text { De qui dépendez-vous pour accéder à cette ressource, pour } \\
\text { pouvoir l'utiliser utilement? Qui contrôle cette ressource? }\end{array}$ & & & \\
\hline 3. & $\begin{array}{l}\text { Quelle est l'importance de cette ressource pour vous? } \\
\text { (sur l'échelle } 0 \ldots 10) ?\end{array}$ & & & \\
\hline \multicolumn{5}{|c|}{$\begin{array}{l}\text { Quel est le comportement de la personne qui contrôle cette ressource qui serait pour vous : } \\
\qquad \begin{array}{c}(a, b, c \text { servent à déterminer les courbes des fonctions d'effet, } \\
\text { d permet de valider les résultas de simulation) }\end{array}\end{array}$} \\
\hline 4. & a- le pire cas (caractérisez ce comportement) & & & \\
\hline 5. & $\begin{array}{l}\text { quel est l'effet de ce comportement sur votre capacité à } \\
\text { atteindre vos objectifs (sur l'échelle }-10 \ldots 0 \ldots+10)\end{array}$ & & & \\
\hline 6. & b- le meilleur cas (caractérisez ce comportement) & & & \\
\hline 7. & $\begin{array}{l}\text { quel est l'effet de ce comportement sur votre capacité à } \\
\text { atteindre vos objectifs (sur l'échelle }-10 \ldots 0 \ldots+10)\end{array}$ & & & \\
\hline 8. & $\begin{array}{l}\text { c- le cas neutre, ni favorable (facilitant) ni défavorable } \\
\text { (pénalisant) (caractérisez ce comportement) }\end{array}$ & & & \\
\hline 9. & $\begin{array}{l}\text { quel est l'effet de ce comportement sur votre capacité à } \\
\text { atteindre vos objectifs (sur l'échelle }-10 \ldots 0 \ldots+10)\end{array}$ & & & \\
\hline 10. & $\begin{array}{l}\text { d- le cas que vous rencontrez habituellement? (caractérisez } \\
\text { ce comportement) }\end{array}$ & & & \\
\hline 11. & $\begin{array}{l}\text { quel est l'effet de ce comportement sur votre capacité à } \\
\text { atteindre vos objectifs (sur l'échelle }-10 \ldots 0 \ldots+10)\end{array}$ & & & \\
\hline 12. & $\begin{array}{l}\begin{array}{l}\text { Qui compte pour vous, de qui êtes-vous solidaire } \\
\text { opposé ? } \\
\text { comme partenaire (sur l'échelle } 0 \ldots 10) \\
\text { comme adversaire (sur l'échelle }-10 \ldots 0)\end{array}\end{array}$ & & & \\
\hline
\end{tabular}

Citation de l'article : Sibertin-Blanc C., 2018. Une analyse formelle du jeu des acteurs autour du projet de barrage de Sivens. Nat. Sci. Soc. 26, 3, 291-307. 